\title{
Population densities in a titanium hollow cathode
}

\author{
K Danzmann and M Kock \\ Institut für Plasmaphysik, Universität Hannover, 3000 Hannover, Callinstrasse 38, West \\ Germany
}

Received 30 December 1980, in final form 10 April 1981

\begin{abstract}
Measurements are reported of population densities in the energy level systems of neutral and singly ionised titanium. The discharge under investigation was a high-current hollow cathode operated in argon or neon as a buffer gas. The measured kinetic and population temperatures of the neutral species differ by more than a factor of two. For the metal ions there is direct evidence of the effects of charge transfer and Penning collisions producing departures from a Boltzmann distribution. For the Ar-Ti hollow-cathode system inversion between the populations of the $5 \mathrm{~s}$ and $5 \mathrm{p}$ configurations has been found.
\end{abstract}

\section{Introduction}

The development of gas discharges for laser applications based on charge transfer as the inversion mechanism has renewed the interest in hollow cathodes (e.g. McNeil et al 1976). A large number of metal-noble-gas combinations are promising candidates for laser oscillation in the ultraviolet (Johansson and Litzén 1980). It would be desirable for the selection and optimisation of these systems to have a comprehensive theoretical model for this type of discharge. Rather successful attempts have been made to develop a semi-empirical model describing the dependence of the metal-vapour and noble-gas ion densities on the current (Warner et al 1979). For the development of a complete model one needs information about the distribution functions of the level populations of the atomic and ionic metal species. Until now such information has been available only on a rather indirect basis:

(i) by observing the output power, if laser action has already been attained, or

(ii) in the form of line enhancement measurements by comparing spectra of continuous and pulsed hollow-cathode discharges (Johansson and Litzén 1978).

We report measurements of absolute population densities for a number of representative energy levels for neutral and ionised titanium in a high-current hollow cathode with argon and neon as buffer gases.

\section{Experimental}

The hollow-cathode discharge as described by Danzmann and Kock (1980) consisted of two cylindrical anodes symmetrically opposing the cathode with a straight-through cylindrical bore of $100 \mathrm{~mm}$ length and a diameter of $8 \mathrm{~mm}$. After evacuation by means of a turbomolecular pump down to a pressure of $10^{-6} \mathrm{mbar}$ the discharge was operated 
in $99.9995 \%$ pure argon at a pressure of $0.6 \mathrm{mbar}$ or in neon at a pressure of 7 mbar. These pressures correspond to the most uniform discharges. Discharge currents of up to $20 \mathrm{~A}$ were supplied by a $600 \mathrm{~V}, 150 \mathrm{~A}$ power supply with an RMS ripple of $2 \%$.

The population densities of neutral and ionised titanium in the ground level and in the metastable levels have been determined by means of the hook method (e.g. Marlow 1967). The advantages over conventional absorption spectroscopy are as follows:

(i) no intensity measurements are required;

(ii) the optical thickness of the lines does not enter;

(iii) the hook spectra can be recorded on film using its immense storage capacity, whereas the non-linear response of a film is of no influence.

The apparatus consisted of a $3.4 \mathrm{~m}$ Ebert spectrograph, a Michelson interferometer and a high-power capillary discharge serving as a background light source. The hook spectra were evaluated using the hook technique (e.g. Huber 1971) and with hook vernier (Sandeman 1979).

In hollow cathodes population densities of excited levels are too small for application of the hook method. We determined the corresponding population densities by emission spectroscopy employing a $2 \mathrm{~m} \mathrm{McPherson} \mathrm{monochromator} \mathrm{in} \mathrm{conjunction}$ with a photon-counting system. The line intensities were calibrated with a carbon arc as a radiation standard using the absolute data given by Einfeld and Stuck (1978) and by Magdeburg and Schley (1966). The optical thickness of the lines was checked in a way similar to that described in an earlier paper (Danzmann and Kock 1980). For the emission measurements, a length of plasma lying within $1 \mathrm{~mm}$ of the axis of the hollow cathode (where the intensity is most uniform) was imaged 'end on' onto the monochromator. Due to the lack of spatial resolution in the axial direction the densities measured represent values averaged over the whole length of the column.

For the evaluation of the experimental data we have used the oscillator strengths of Danzmann and Kock (1980) and Kühne et al (1978). For some lines we used the data of Roberts et al (1975) and Wiese and Fuhr (1975).

\section{Results and discussion}

Results for $\mathrm{Ti} \mathrm{I}$ are shown in figures 1 and 2. The population densities of the excited levels of Ti i clearly follow a Boltzmann distribution with a corresponding population temperature of $4900 \mathrm{~K}$ for the $\mathrm{Ti}-\mathrm{Ar}$ system and $4700 \mathrm{~K}$ for the $\mathrm{Ti}-\mathrm{Ne}$ system. In the current range investigated these temperatures are independent of the discharge current. The ground state and the metastable states are overpopulated with respect to this Boltzmann distribution. The overpopulation decreases with increasing current.

We have also determined the kinetic temperature of the metallic component from the half widths of the Gaussian line profiles obtained with a high-resolution FabryPerot interferometer. The atom and ion kinetic temperature is $2000 \mathrm{~K} \pm 8 \%$ in the $\mathrm{Ti}-\mathrm{Ne}$ hollow-cathode discharge in accordance with the results Vaessen et al (1978) have obtained from a $\mathrm{Cu}-\mathrm{Ne}$ hollow cathode laser. Gerstenberger et al (1980) found close agreement between the kinetic temperature and the population temperature of some highly excited $\mathrm{He}$ I levels in a $\mathrm{He}-\mathrm{Cu}$ hollow cathode, both of which were of the order of $1000 \mathrm{~K}$. Those authors assumed the population temperature of the neutral species (at least for the highly excited levels) to be equal to the electron temperature of the low energy group of electrons in a hollow cathode. In the $\mathrm{Ti}-\mathrm{Ne}$ cathode, however, the kinetic and population temperatures differ by more than a factor of two. 


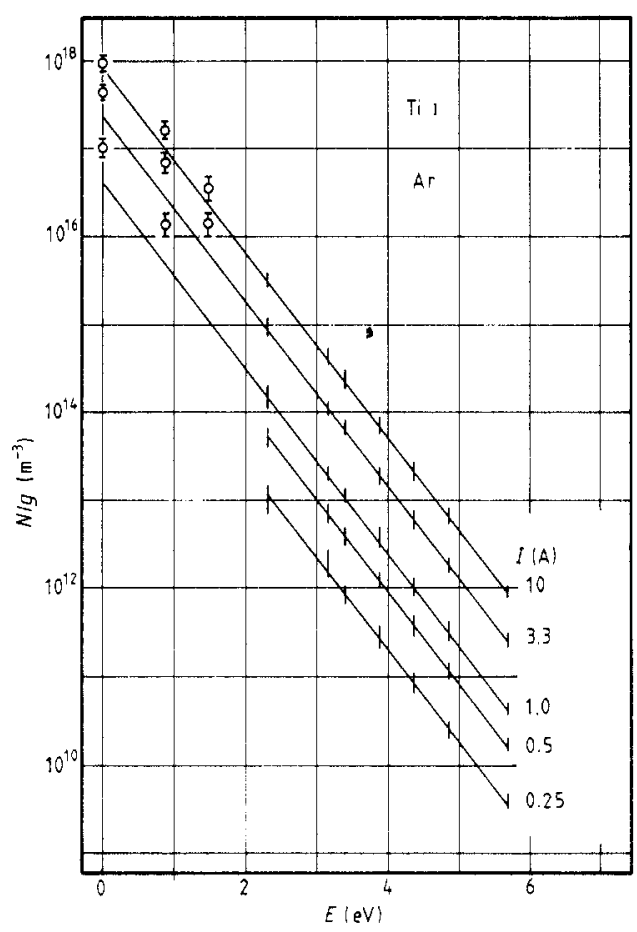

Figure 1. Population densities, except for the statistical weight factor, as a function of level energy for neutral titanium (Boltzmann plot), with the discharge current as a parameter. Buffer gas is argon at a pressure of 0.6 mbar. Error bars with open circles denote results from hook measurements, solid error bars those from emission measurements.

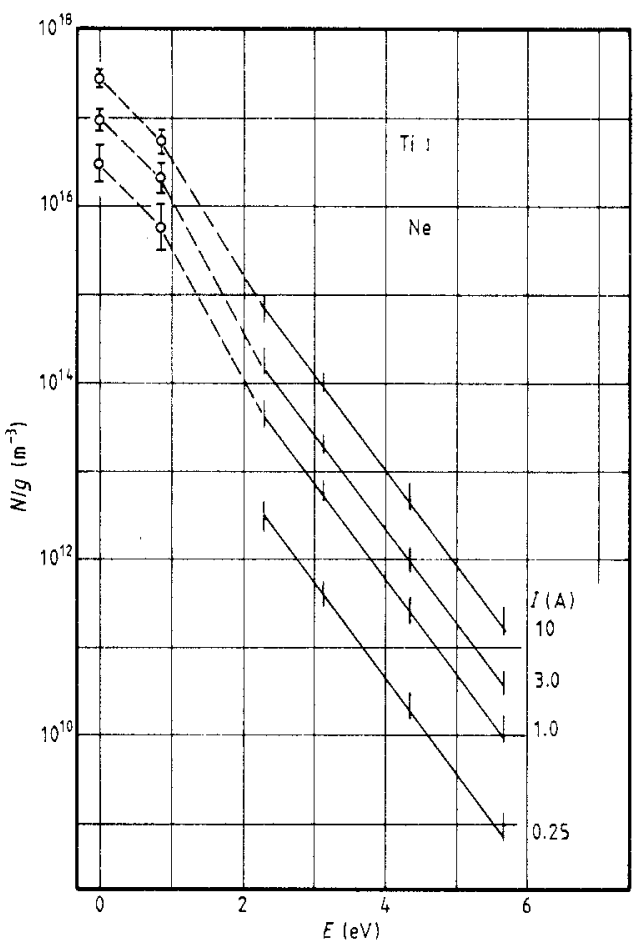

Figure 2. Population densities for neutral titanium with neon as a buffer gas at a pressure of 7 mbar.

Moreover the population temperature of neutral titanium is higher by nearly a factor of five than the $\mathrm{He}$ i population temperature measured by those authors.

In our opinion the population temperature is not likely to be equal to the electron temperature, although it is felt that the energy levels of the neutrals are populated by electron collisions. Excitation and de-excitation collisions may be in equilibrium, but depopulation of excited levels by ionising collisions cannot be balanced on a local basis alone. Under the present discharge conditions three-body recombination is a very improbable process and rates for radiative recombination are too low by several orders of magnitude. Diffusion to the wall should be the dominant ion loss mechanism. Estimates show that the electron temperature may be substantially higher than the population temperature.

The population distribution of ionised titanium (figure 3) shows a completely different behaviour. A description by means of a Boltzmann distribution is no longer possible. The population density as a function of level energy passes through a minimum and rises again, so that the populations of the high-lying levels show inversion. Evidently these levels are populated to a considerable extent via the thermal energy charge transfer reaction

$$
\mathrm{Ar}^{+}+\mathrm{Ti} \rightarrow \mathrm{Ar}+\mathrm{Ti}^{+*} \text {. }
$$




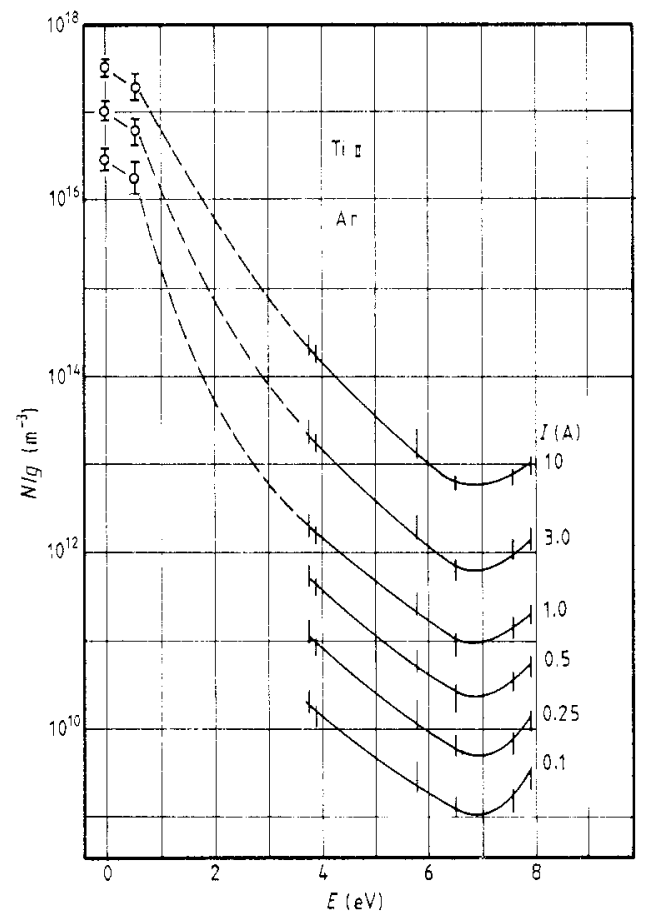

Figure 3. Population densities for ionised titanium with argon as a buffer gas at a pressure of 0.6 mbar.

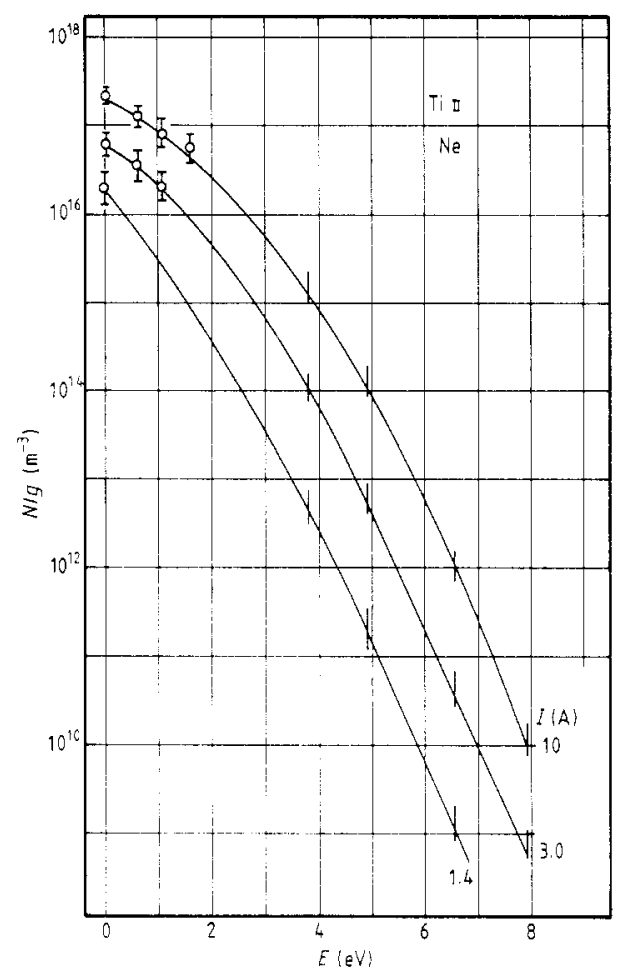

Figure 4. Population densities for ionised titanium with neon as a buffer gas at a pressure of 7 mbar.

Littlewood et al (1979) have measured the production rates of zinc, cadmium and mercury ions in their ground states relative to those in their excited states in charge transfer collisions with $\mathrm{He}^{+}$. They found the cross sections for ground-state production to be very small. Thus these collisions will selectively populate levels with an energy defect of less than $0.5 \mathrm{eV}$.

The inverted levels are situated $15.5 \mathrm{eV}$ above the Ti I ground state which is $0.2 \mathrm{eV}$ less than the ionisation energy of argon. Unfortunately we could not determine the population densities of levels still higher in energy, as the oscillator strengths of the lines of interest are not known.

The measurements presented explain the results of Johansson and Litzén (1980) who observed strong enhancement of intensities belonging to the $5 \mathrm{~s}-5 \mathrm{p}$ transition scheme in $\mathrm{Ti}$ II. The level energies pertaining to these configurations are situated in the minimum and in the rising parts of the population distribution. It may be seen from figure 3 that the degree of inversion decreases slightly with increasing current. A possible explanation for this behaviour could be the fact that the noble-gas ion density would saturate with increasing current and thus ionisation by collisions with fast electrons accelerated in the cathode fall would make an increasingly important contribution. Another reason could be de-excitation of the inverted levels by collisions of the second kind with thermalised electrons. Since we have no direct information about the electron temperature we cannot yet decide which mechanism is the dominant one.

The ionic level populations show a completely different distribution if neon is used as a buffer gas instead of argon. No inversion can be seen from figure 4 ; on the contrary 
all the levels with moderate energies are overpopulated in comparison with a Boltzmann distribution. The relative population temperature of the low-lying levels increases with increasing current. This form of population distribution depends on Penning ionisation between neon metastables and titanium neutrals

$$
\mathrm{Ne}^{*}+\mathrm{Ti} \rightarrow \mathrm{Ne}+\mathrm{Ti}^{+*}+\mathrm{e} .
$$

Although the energy of the neon metastables is nearly equal to the ionisation energy of argon, $\mathrm{Ti}$ in levels corresponding to this energy are not overpopulated. Levels with energy defects of up to several $\mathrm{eV}$ can be populated by Penning collision processes which do not require close energy resonances, as was pointed out by Green and Webb (1974).

\section{Conclusion}

The significance of the present investigation is the experimental determination of absolute population density distributions in a hollow cathode as a function of the discharge current. We have chosen titanium as the cathode material for our investigation because the oscillator strengths required in the evaluation are very well known for both neutral and ionised titanium. Similar investigations for all other noble-gas-metal combinations are possible, depending on the availability of $f$ values. Such measurements would contribute to the development of numerical models for hollow-cathode discharges.

\section{Acknowledgments}

Part of the work has been supported by Deutsche Forschungsgemeinschaft (DFG). The authors are indebted to E Schulz-Gulde for valuable comments. The technical assistance of L Borth, K Witkop, and G Dietrich is gratefully acknowledged.

\section{References}

Danzmann K and Kock M 1980 J. Phys. B: At. Mol. Phys. 13 2051-9

Einfeld D and Stuck D 1978 Z. Naturf. 33a 502-4

Gerstenberger D C, Solanki R and Collins G J 1980 IEEE J. Quant. Electron. QE-16 820-34

Green J M and Webb C E 1974 J. Phys. B: At. Mol. Phys. 7 1698-711

Huber M C E 1971 Modern Optical Methods in Gas Dynamic Research ed D S Donsanjh (New York: Plenum) pp 85-112

Johansson S and Litzén U 1978 J. Phys, B: At. Mol. Phys. 11 L703-6

1980 J. Phys. B: Ai. Mol, Phys. 13 L253-6

Kühne M, Danzmann K and Kock M 1978 Astron. Astrophys. 64 111-3

Littlewood I M, Piper J A and Webb C E 1979 J. Phys. B: At. Mol. Phys. 12 1399-405

McNeil J R, Collins G J, Persson K B and Franzen D L 1976 Appl. Phys. Lett. 28 207-9

Magdeburg $\mathrm{H}$ and Schley U 1966 Z. Angew. Phys. $20465-73$

Marlow W G 1967 Appl. Opt. 6 1715-24

Roberts J R, Voigt P A and Czernichowski A 1975 Astrophys. J. 197 791-8

Sandeman R J 1979 Appl._Opt. 18 3873-4

Vaessen P H M, DeHoog F J and McNeil J R 1978 Phys. Lett. 68A 204-6

Warner B E, Persson K B and Collins G J 1979 J. Appl. Phys. 50 5694-703

Wiese W L and Fuhr J R 1975 J. Phys. Chem. Ref. Data 4 263-333 